TEMA Tend. Mat. Apl. Comput., 13, No. 2 (2012), 167-178.

doi: $10.5540 /$ tema.2012.013.02.0167

(C) Uma Publicação da Sociedade Brasileira de Matemática Aplicada e Computacional.

\title{
Uma Nova Classe de Distribuições Generalizadas
}

J.A. RODRIGUES, L.M. CHAVES ${ }^{1}$, Departamento de Ciências Exatas, DEX, UFLA - Universidade Federal de Lavras, 37200-000 Lavras, MG, Brasil.

F. CASTELLARES 2 , Departamento de Estatística, ICEX, UFMG - Universidade Federal de Minas Gerais, 31270-901 Belo Horizonte, MG, Brasil.

\begin{abstract}
Resumo. Neste trabalho, é introduzida uma família de distribuições denominada hipergeométrica confluente G que inclui os importantes modelos: beta normal, beta Weibull, beta Gumbel, beta Pareto, entre outros. Novas distribuições são apresentadas como membros dessa família, por exemplo, a distribuição hipergeométrica confluente normal e a distribuição hipergeométrica confluente Weibull. A estimação dos parâmetros dessa nova classe de distribuições generalizadas é estudada utilizando o Método da Máxima Verossimilhança e sua potencialidade é demonstrada na modelagem de um conjunto de dados reais de trinta e cinco crianças com deficiência do hormônio de crescimento.
\end{abstract}

Palavras-chave. Distribuição beta generalizada, distribuição hipergeométrica confluente, Método da Máxima Verossimilhança.

\section{Introdução}

Nos últimos anos, têm-se buscado novas distribuições para modelagem do tempo de duração de componentes ou tempo de vida de indivíduos. Esse fato é justificado, em função das distribuições existentes, muitas vezes não se ajustarem de forma satisfatória ao conjunto de dados reais em estudo. Nesse contexto, generalizações das distribuições existentes têm sido propostas. Algumas dessas distribuições são: a beta Pareto apresentada por [1], a Weibul exponenciada estudada por [2, 9, 10, 13, 15], a beta normal estudada por [4], a beta Weibull inversa introduzida por [6], a beta Weibull proposta por [7], a beta Gumbel proposta por [11], a beta exponencial estudada por [12], a beta Burr XII inserida por [16], a beta Weibull modificada estudada por [19] e a Gumbel exponenciada tratada por [18].

O objetivo deste trabalho é introduzir uma nova classe de distribuições, denominada hipergeométrica confluente $\mathrm{G}$, que generaliza a família de distribuições beta G. Algumas novas distribuições são apresentadas como membros dessa família. A

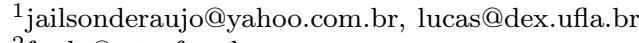

2 fredy@est.ufmg.br
} 
estimação de parâmetros é discutida via Método de Máxima Verossimilhança. Alguns casos especiais são discutidos e uma aplicação a dados reais demonstra sua potencialidade.

O texto é organizado da seguinte forma: na Seção 2 a família hipergeométrica confluente $\mathrm{G}$ é definida e alguns submodelos são discutidos na Seção 3. A estimação dos parâmetros via método da máxima verossimilhança é discutida na Seção 4 . Na Seção 5 é feita a aplicação da distribuição hipergeométrica confluente G na análise de dados reais de trinta e cinco crianças com deficiência do hormônio de crescimento.

Os cálculos envolvidos incluem o uso de algumas funções especiais como a função gama,

$$
\Gamma(a)=\int_{0}^{\infty} t^{\alpha-1} \exp (-t) d t,
$$

a função gama incompleta,

$$
\gamma(a, x)=\int_{0}^{x} t^{\alpha-1} \exp (-t) d t,
$$

a função beta,

$$
B(a, b)=\int_{0}^{1} t^{a-1}(1-t)^{b-1} d t,
$$

e a função hipergeométrica confluente,

$$
{ }_{1} F_{1}(a, b ; x)=\sum_{i=0}^{\infty} \frac{(a)_{i}}{(b)_{i} i !} x^{i},
$$

sendo que $(z)_{i}=(z)(z+1) \ldots(z+i-1)$ denota o fatorial ascendente. As propriedades dessas funções especiais podem ser vistas em [14].

\section{O Modelo}

Considere a distribuição hipergeométrica confluente, ver [5], com função densidade de probabilidade (fdp),

$$
f(x)=\frac{x^{a-1}(1-x)^{b-1} \exp (-c x)}{B(a, b){ }_{1} F_{1}(a, a+b,-c)},
$$

em que $0<x<1, a>0, b>0,-\infty<c<+\infty$.

Combinando os trabalhos de [3], [4] e [17] pode-se construir uma nova classe de distribuições, denominada hipergeométrica confluente G. Se $G(x)$ denota uma função de distribuição (fd) de uma variável aleatória em que $x \in I$, então, a fd hipergeométrica confluente $\mathrm{G}$ é dada por,

$$
F(x)=\frac{1}{B(a, b){ }_{1} F_{1}(a, a+b,-c)} \int_{0}^{G(x)} t^{a-1}(1-t)^{b-1} \exp (-c t) d t, \quad x \in I,
$$


a fdp associada a essa distribuição é,

$$
f(x)=\frac{g(x) G(x)^{a-1}[1-G(x)]^{b-1} \exp [-c G(x)]}{B(a, b){ }_{1} F_{1}(a, a+b ;-c)}, \quad g(x)=\frac{d G(x)}{d x},
$$

a função de sobrevivência é,

$$
S(x)=1-\frac{1}{B(a, b){ }_{1} F_{1}(a, a+b,-c)} \int_{0}^{G(x)} t^{a-1}(1-t)^{b-1} \exp (-c t) d t,
$$

e a função taxa de falha é dada por,

$$
\lambda(x)=\frac{g(x) G(x)^{a-1}(1-G(x))^{b-1} \exp [-c G(x)]}{B(a, b){ }_{1} F_{1}(a, a+b ;-c)-\int_{0}^{G(x)} t^{a-1}(1-t)^{b-1} \exp (-c t) d t} .
$$

Claramente quando $a=b=1$ e $c=0$ o modelo hipergeométrico confluente $\mathrm{G}$ reduz-se a distribuição original $G(x)$.

Se $1>|z|$ e se $b$ é um número real positivo não inteiro, então

$$
(1-z)^{b-1}=\sum_{j=0}^{\infty} \frac{(-1)^{j} \Gamma(b) z^{j}}{\Gamma(b-j) j !}
$$

Utilizando a expansão em série (2.6), a fd hipergeométrica confluente G pode ser expressa em termos da função gama incompleta,

$$
F(x)=\frac{\Gamma(b)}{B(a, b){ }_{1} F_{1}(a, a+b ;-c)} \sum_{j=0}^{\infty} \frac{(-1)^{j} \gamma(a+j, c G(x))}{c^{a+j} \Gamma(b-j) j !} .
$$

Se $b>0$ é um número inteiro, o índice $j$ varia de zero até $b-1$.

\section{Alguns Casos Especiais}

Nesta seção, são tratados alguns exemplos da distribuição hipergeométrica confluente G. Esses submodelos generalizam diversas distribuições importantes existentes na literatura; como é o caso da beta normal e da beta Weibull.

\section{- Distribuição hipergeométrica confluente normal}

Ao substituir $G(x)$ e $g(x)$ em (2.3) pelas respectivas fd e fdp da distribuição normal $N\left(\mu, \sigma^{2}\right)$ obtemos, a fdp hipergeométriica confluente normal,

$$
f(x)=\frac{\phi\left(\frac{x-\mu}{\sigma}\right) \Phi\left(\frac{x-\mu}{\sigma}\right)^{a-1}\left[1-\Phi\left(\frac{x-\mu}{\sigma}\right)\right]^{b-1} \exp \left[-c \Phi\left(\frac{x-\mu}{\sigma}\right)\right]}{B(a, b)_{1} F_{1}(a, a+b ;-c)},
$$

sendo que $-\infty<x<+\infty, a>0, b>0, \sigma>0,-\infty<c<+\infty,-\infty<\mu<$ $+\infty, \phi(\cdot)$ é a fdp normal padrão e $\Phi(\cdot)$ é a fd normal padrão. Uma variável aleatória $X$ com fdp (3.1) será denotada por $X \sim H C N\left(a, b, c, \mu, \sigma^{2}\right)$. Caso, $c=0$ a distribuição $H C N\left(a, b, 0, \mu, \sigma^{2}\right)$ corresponde ao modelo beta normal, proposto por [4] e a distribuição $\operatorname{HCN}\left(1,1,0, \mu, \sigma^{2}\right)$ coincide com o modelo normal. 


\section{- Distribuição hipergeométrica confluente log-normal}

Quando $G(x)$ e $g(x)$ em (2.3) são substituídas pelas respectivas fd e fdp da distribuição log-normal $L N\left(\mu, \sigma^{2}\right)$ obtém-se, a fdp hipergeométriica confluente log-normal,

$f(x)=\frac{\phi\left(\frac{\log x-\mu}{\sigma}\right) \Phi\left(\frac{\log x-\mu}{\sigma}\right)^{a-1}\left[1-\Phi\left(\frac{\log x-\mu}{\sigma}\right)\right]^{b-1} \exp \left[-c \Phi\left(\frac{\log x-\mu}{\sigma}\right)\right]}{B(a, b)_{1} F_{1}(a, a+b ;-c)}$,

sendo que $x>0, a>0, b>0, \sigma>0,-\infty<c<+\infty,-\infty<\mu<+\infty$, $\phi(\cdot)$ é a fdp normal padrão e $\Phi(\cdot)$ é fd normal padrão. Caso, $c=0$, tem-se o modelo beta log-normal, para $a=b=1$ e $c=0$, obtém-se a distribuição log-normal. Uma variável aleatória $X$ com densidade (3.2) será indicada, $X \sim H C L N\left(a, b, c, \mu, \sigma^{2}\right)$.

\section{- Distribuição hipergeométrica confluente Weibull}

A distribuição Weibull que inclui os submodelos exponencial e Rayleigh é uma das mais importantes da estatística. A fd e a fdp desse modelo são respectivamente,

$$
G(x)=1-\exp \left[-(s x)^{k}\right]
$$

$$
g(x)=k s^{k} x^{k-1} \exp \left[-(s x)^{k}\right]
$$

em que $x>0, k>0$ e $s>0$. Dessa forma, a densidade hipergeométriica confluente Weibull, denotada por $H C W(a, b, c, k, s)$, pode ser expressa como,

$$
f(x)=\frac{k s^{k} x^{k-1}\left\{1-\exp \left[-(s x)^{k}\right]\right\}^{a-1} \exp \left\{c \exp \left[-(s x)^{k}\right]-b(s x)^{k}\right\}}{\exp (c) B(a, b)_{1} F_{1}(a, a+b ;-c)} .
$$

Essa distribuição inclui, quando $c=0$, o modelo beta Weibull descrita por [7]. Se $k=1$ juntamente com $c=0$, tem-se o modelo beta exponencial proposto por [12]. Caso, $b=1$ e $c=0$ origina-se o modelo bastante conhecido Weibull exponenciado. Se $k=2$ e $c=0$ origina-se a distribuição beta Rayleigh. Se $a=b=1$ e $c=0$, obtém-se o modelo Weibull. Se $a=b=1, c=0$ e em adicional $k=1$ resulta no modelo exponencial.

- Distribuição hipergeométrica confluente gama Seja $Y$ uma variável aleatória com fd, $G(y)=\gamma(\alpha, \beta y) / \Gamma(\alpha)$ em que $y>0, \alpha>0$ e $\beta>0$. A fdp de uma variável aleatória $X$ com distribuição hipergeométriica confluente gama, indicada por $X \sim H C G(a, b, c, \alpha, \beta)$, pode ser escrita como,

$$
f(x)=\frac{\beta^{\alpha} x^{\alpha-1} \gamma(\alpha, \beta x)^{a-1}[\Gamma(\alpha)-\gamma(\alpha, \beta x)]^{b-1}}{B(a, b){ }_{1} F_{1}(a, a+b ;-c) \Gamma(\alpha)^{a+b-1}} \exp \left[-\beta x-c \frac{\gamma(\alpha, \beta x)}{\Gamma(\alpha)}\right] .
$$

Se $c=0$, a fdp (3.6) reduz-se a fdp do modelo beta gama. 


\section{- Distribuição hipergeométrica confluente Pareto}

Se $X$ é uma variável aleatória Pareto com fd, $G(x)=1-(x / s)^{-k}$ em que $x \geq s$, $k>0$ e $s>0$. A densidade de uma variável aleatória $X$ com distribuição hipergeométriica confluente Pareto, indicada por $X \sim \operatorname{HCP}(a, b, c, k, s)$, pode ser expressa como,

$$
f(x)=\frac{k s^{b k} x^{-(b k+1)}\left[1-(x / s)^{-k}\right]^{a-1} \exp \left[c(x / s)^{-k}\right]}{\exp (c) B(a, b)_{1} F_{1}(a, a+b ;-c)} .
$$

A distribuição beta Pareto introduzida por [1] é um importante caso particular de (3.7) quando $c=0$.

\section{- Distribuição hipergeométrica confluente Gumbel}

A fd e a fdp do modelo Gumbel são dadas, respectivamente, por:

$$
G(x)=\exp \left[-\exp \left(-\frac{x-\mu}{\sigma}\right)\right]
$$

e

$$
g(x)=\frac{1}{\sigma} \exp \left[-\frac{x-\mu}{\sigma}-\exp \left(-\frac{x-\mu}{\sigma}\right)\right]
$$

com $-\infty<x<+\infty,-\infty<\mu<+\infty$ e $\sigma>0$. O modelo hipergeométrico confluente Gumbel tem fdp dada por,

$$
f(x)=\frac{[1-\exp (-u)]^{b-1} \exp \{-u-a \exp (-u)-c \exp [-\exp (-u)]\}}{\sigma B(a, b){ }_{1} F_{1}(a, a+b ;-c)},
$$

sendo que $u=(x-\mu) / \sigma$. A distribuição beta Gumbel, ver [11], é um submodelo de (3.10) quando $c=0$. Caso $b=1$ juntamente com $c=0$, tem-se a fdp Gumbel exponenciada estudada. Uma variável aleatória $X$ com fdp (3.10) será denotada por $X \sim \operatorname{HCGB}(a, b, c, \mu, \sigma)$.

\section{- Distribuição hipergeométrica confluente Weibull inversa}

A fd da distribuição Weibull inversa é definida como $G(x)=\exp \left[-(s / x)^{k}\right]$ em que $x>0, k>0$ e $s>0$. Dessa forma, a fdp da distribuição hipergeométriica confluente Weibull inversa, indicada por $X \sim H C W I(a, b, c, k, s)$, pode ser expressa como,

$$
f(x)=\frac{k s^{k}\left\{1-\exp \left[-(s / x)^{k}\right]\right\}^{b-1} \exp \left\{-a(s / x)^{k}-c \exp \left[-(s / x)^{k}\right]\right\}}{x^{k+1} B(a, b){ }_{1} F_{1}(a, a+b ;-c)} .
$$

Se $c=0$, a fdp (3.11) reduz-se ao modelo beta Weibull inversa. Caso $b=1 \mathrm{e}$ $c=0$, tem-se a distribuição Weibull inversa exponenciada.

Nas Figuras de 1 a 7 são exibidas algumas das formas possíveis das fdp para os casos especiais da distribuição hipergeométrica confluente G. 


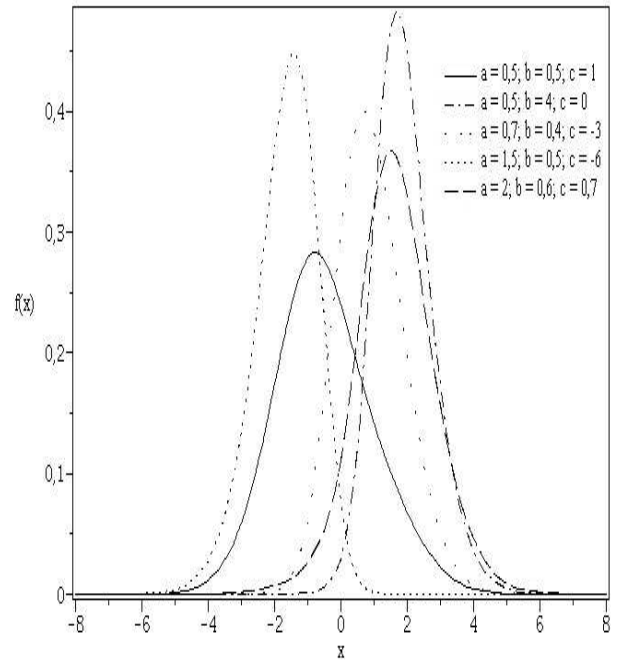

Figura 1: Funções densidade de probabilidade $H C N(a, b, c, 0,1)$.

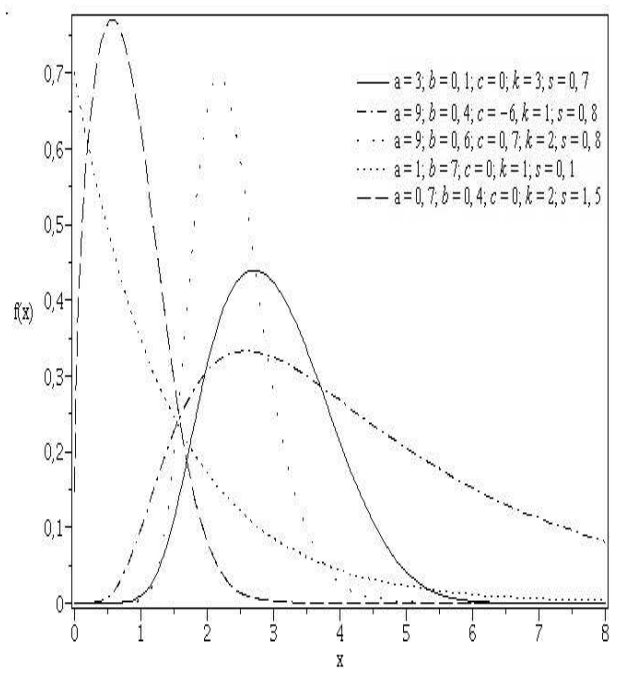

Figura 3: Funções densidade de probabilidade $H C W(a, b, c, k, s)$.

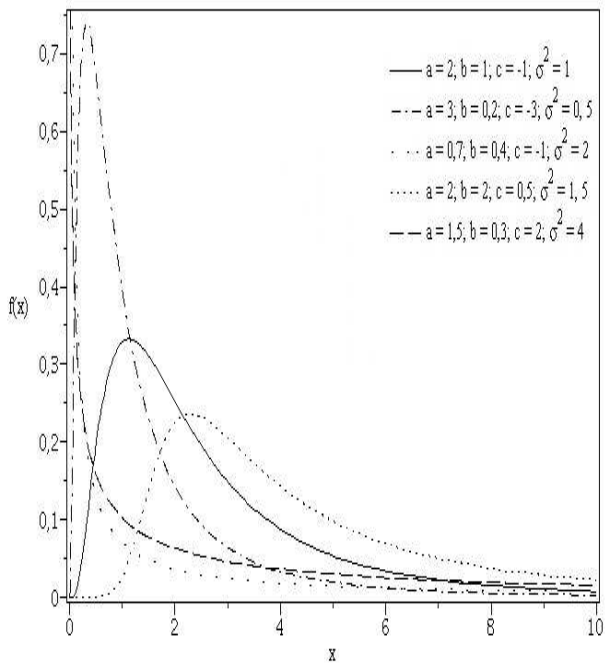

Figura 2: Funções densidade de probabilidade $\operatorname{HCLN}\left(a, b, c, 0, \sigma^{2}\right)$.

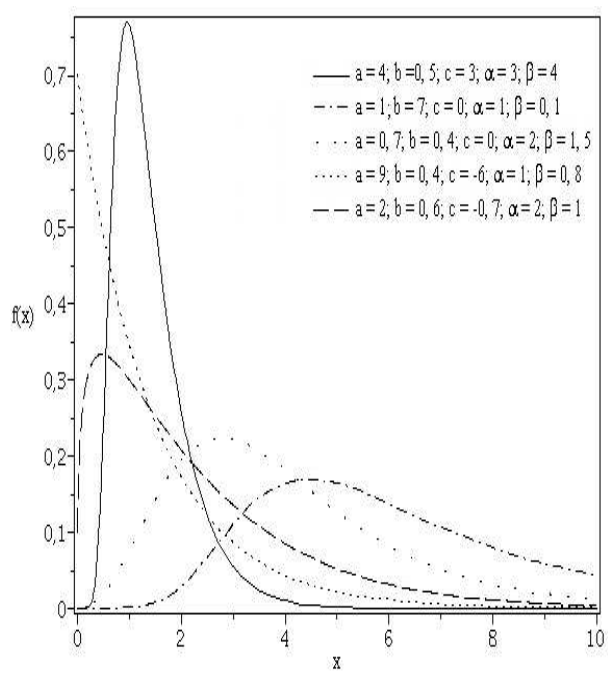

Figura 4: Funções densidade de probabilidade $H C G(a, b, c, \alpha, \beta)$.

\section{Estimação dos Parâmetros}

Seja $X_{1}, \ldots, X_{n}$ uma amostra aleatória da distribuição hipergeométrica confluente $\mathrm{G}$ com vetor paramétrico $\widetilde{\theta}=(a, b, c, \widetilde{\eta})$. O vetor $p$-dimensional de parâmetros 


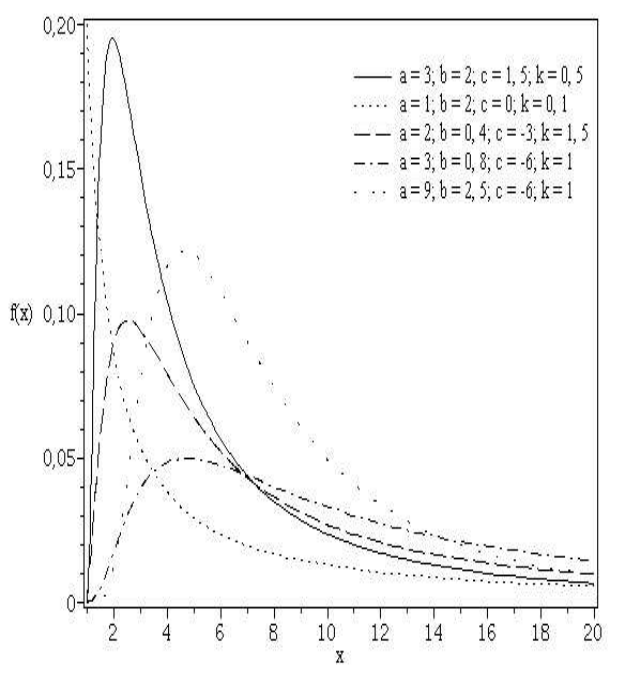

Figura 5: Funções densidade de probabilidade $H C P(a, b, c, k, 1)$.

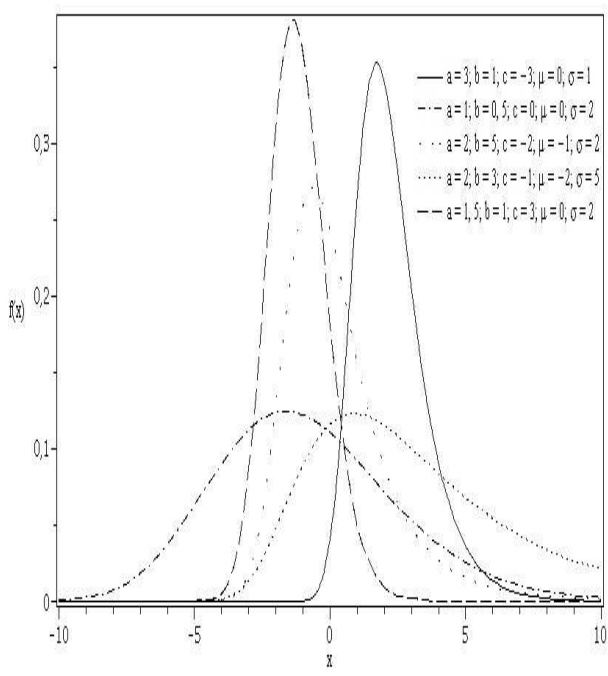

Figura 6: Funções densidade de probabilidade $\operatorname{HCGB}(a, b, c, \mu, \sigma)$.

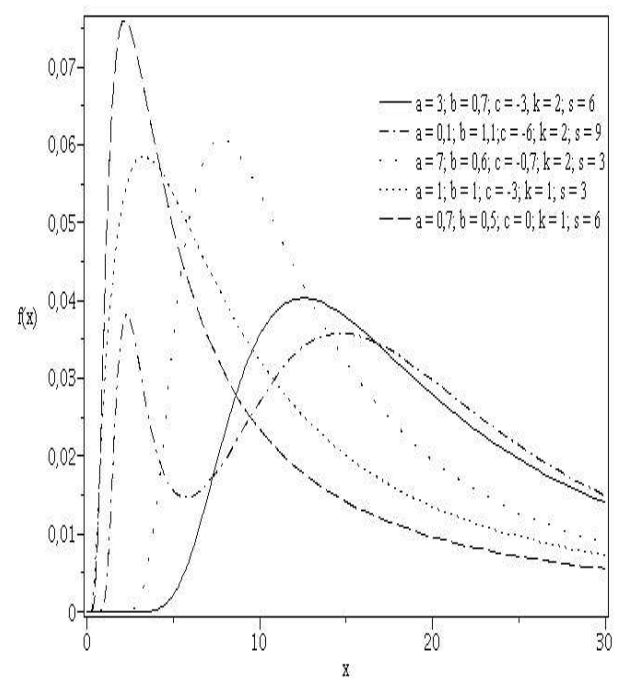

Figura 7: Funções densidade de probabilidade $H C W I(a, b, c, k, s)$. 
$\widetilde{\eta}=\left(\eta_{1}, \ldots, \eta_{p}\right)$ é proveniente da distribuição geradora $G(x ; \widetilde{\eta})$. A função de verossimilhança para o vetor paramétrico $\widetilde{\theta}$ pode ser escrita como,

$$
L(\widetilde{\theta})=\frac{\exp \left[-c \sum_{i=0}^{n} G\left(x_{i} ; \widetilde{\eta}\right)\right]}{B(a, b)^{n}{ }_{1} F_{1}(a, a+b ;-c)^{n}} \prod_{i=1}^{n} g\left(x_{i} ; \widetilde{\eta}\right) G\left(x_{i} ; \widetilde{\eta}\right)^{a-1}\left[1-G\left(x_{i} ; \widetilde{\eta}\right)\right]^{b-1} .
$$

Enquanto a correspondente função log-verossimilhança pode ser expressa como:

$$
\begin{aligned}
l(\widetilde{\theta})= & -n \log B(a, b)-n \log { }_{1} F_{1}(a, a+b ;-c)-c \sum_{i=0}^{n} G\left(x_{i} ; \widetilde{\eta}\right)+\sum_{i=0}^{n} \log g\left(x_{i} ; \widetilde{\eta}\right) \\
& +(a-1) \sum_{i=0}^{n} \log G\left(x_{i} ; \widetilde{\eta}\right)+(b-1) \sum_{i=0}^{n} \log \left[1-G\left(x_{i} ; \widetilde{\eta}\right)\right]
\end{aligned}
$$

as estimativas de máxima verossimilhança de $a, b, c$ e $\eta_{j}$ são as soluções do sistema de equações não-lineares:

$$
\begin{aligned}
& \frac{\partial l(\widetilde{\theta})}{\partial a}=-n \psi(a)+n \psi(a+b)-n \frac{\partial \log { }_{1} F_{1}(a, a+b ;-c)}{\partial a}+\sum_{i=0}^{n} \log G\left(x_{i} ; \widetilde{\eta}\right)=0 \\
& \frac{\partial l(\widetilde{\theta})}{\partial b}=-n \psi(b)+n \psi(a+b)-n \frac{\partial \log { }_{1} F_{1}(a, a+b ;-c)}{\partial b}+\sum_{i=0}^{n} \log \left[1-G\left(x_{i} ; \widetilde{\eta}\right)\right]=0 \\
& \frac{\partial l(\widetilde{\theta})}{\partial c}=-n \frac{\partial \log { }_{1} F_{1}(a, a+b ;-c)}{\partial c}-\sum_{i=0}^{n} G\left(x_{i} ; \widetilde{\eta}\right)=0 \\
& \frac{\partial l(\widetilde{\theta})}{\partial \eta_{j}}=\sum_{i=0}^{n}\left\{\frac{1}{g\left(x_{i} ; \widetilde{\eta}\right)} \frac{\partial g\left(x_{i} ; \widetilde{\eta}\right)}{\partial \eta_{j}}+\left[\frac{(a-1)}{G\left(x_{i} ; \widetilde{\eta}\right)}+\frac{(1-b)}{1-G\left(x_{i} ; \widetilde{\eta}\right)}-c\right] \frac{\partial G\left(x_{i} ; \widetilde{\eta}\right)}{\partial \eta_{j}}\right\}=0
\end{aligned}
$$

em que $\psi(\cdot)$ denota a função digama $\psi(t)=d \log \Gamma(t) / d t$.

\section{Aplicação}

Nesta seção, foi realizada uma aplicação da família hipergeométrica confluente G, quando $\mathrm{G}$ tem distribuição Weibull.

A distribuição hipergeométrica confluente Weibull foi ajustada utilizando dados do Programa Hormonal de Crescimento da Secretaria da Saúde de Minas Gerais, esses dados são provenientes do tratamento de trinta e cinco crianças com diagnóstico de deficiência do hormônio de crescimento e consistem no tempo até que o medicamento com esse tipo de hormônio atinja o objetivo, Tabela 1. Mais detalhes sobre esse conjunto de dados podem ser encontrados em [8]. 
Tabela 1: Dados do hormônio de crescimento.

\begin{tabular}{ccccccc}
\hline 2,15 & 2,20 & 2,55 & 2,56 & 2,63 & 2,74 & 2,81 \\
2,90 & 3,05 & 3,41 & 3,43 & 3,43 & 3,84 & 4,16 \\
4,18 & 4,36 & 4,42 & 4,51 & 4,60 & 4,61 & 4,75 \\
5,03 & 5,10 & 5,44 & 5,90 & 5,96 & 6,77 & 7,82 \\
8,00 & 8,16 & 8,21 & 8,72 & 10,40 & 13,20 & 13,70 \\
\hline
\end{tabular}

Tabela 2: Estimativa de máxima verossimilhança dos parâmetros e AIC.

\begin{tabular}{lcccccc}
\hline Distribuição & $a$ & $b$ & $c$ & $k$ & $s$ & AIC \\
\hline HC-Weibull & 171,1053 & 0,0814 & $-5,5303$ & 1,2972 & 1,6759 & 163,2685 \\
Beta Weibull & 9,7128 & 0,2510 & $\ldots$ & 1,1877 & 0,8653 & 163,9901 \\
Weibull & $\ldots$ & $\ldots$ & $\ldots$ & 1,9932 & 0,1659 & 168,9772 \\
Exponencial & $\ldots$ & $\ldots$ & $\ldots$ & $\ldots$ & 0,1885 & 188,8149 \\
\hline
\end{tabular}

As estimativas de máxima verossimilhança para o modelo hipergeométrico confluente Weibull e seus submodelos beta Weibull, Weibull e exponencial são exibidas na Tabela 2.

Para selecionar a distribuição com melhor ajuste foi utilizado o critério de informação de Akaike, baseado na teoria de decisão o AIC (Akaike Information Criterion) é definido como a quantidade $A I C=-2 L+2 p$ em que $L$ representa a magnitude máxima da função suporte e $p$ denota o número de parâmetros da distribuição. De acordo com esse critério, o melhor modelo é aquele que apresenta o menor valor do AIC. Na Tabela 2 pode-se observar que a distribuição hipergeométrica confluente Weibull apresentou um ajuste melhor, comparado aos outros modelos probabilísticos alternativos, pois seu AIC apresentou menor valor em relação as outras distribuições consideradas.

A qualidade de ajuste dos modelos também pode ser verificada pelos gráficos de probabilidades. Esses gráficos consistem em representar no plano cartesiano a probabilidade observada versus a probabilidade esperada. Para cada um dos modelos foi plotado $F\left(x_{(i)}\right)$ versus $(i-0,375) /(n+0,25), i=1, \ldots, n$ em que $F(\cdot)$ denota a função de distribuição e $x_{(i)}$ representa os valores amostrais em ordem crescente. Na Figura 8 são exibidos os gráficos de probabilidade as distribuições ajustadas, também por esse critério, a distribuição hipergeométrica confluente Weibull apresentou melhor ajuste em relação as distribuições beta Weibull, Weibull e exponencial.

Comparando as fdp ajustadas com o histograma dos dados observados, Figura 9, novamente pode-se concluir que a distribuição hipergeométrica confluente Weibull oferece um ajuste melhor em relação as outras três distribuições. 

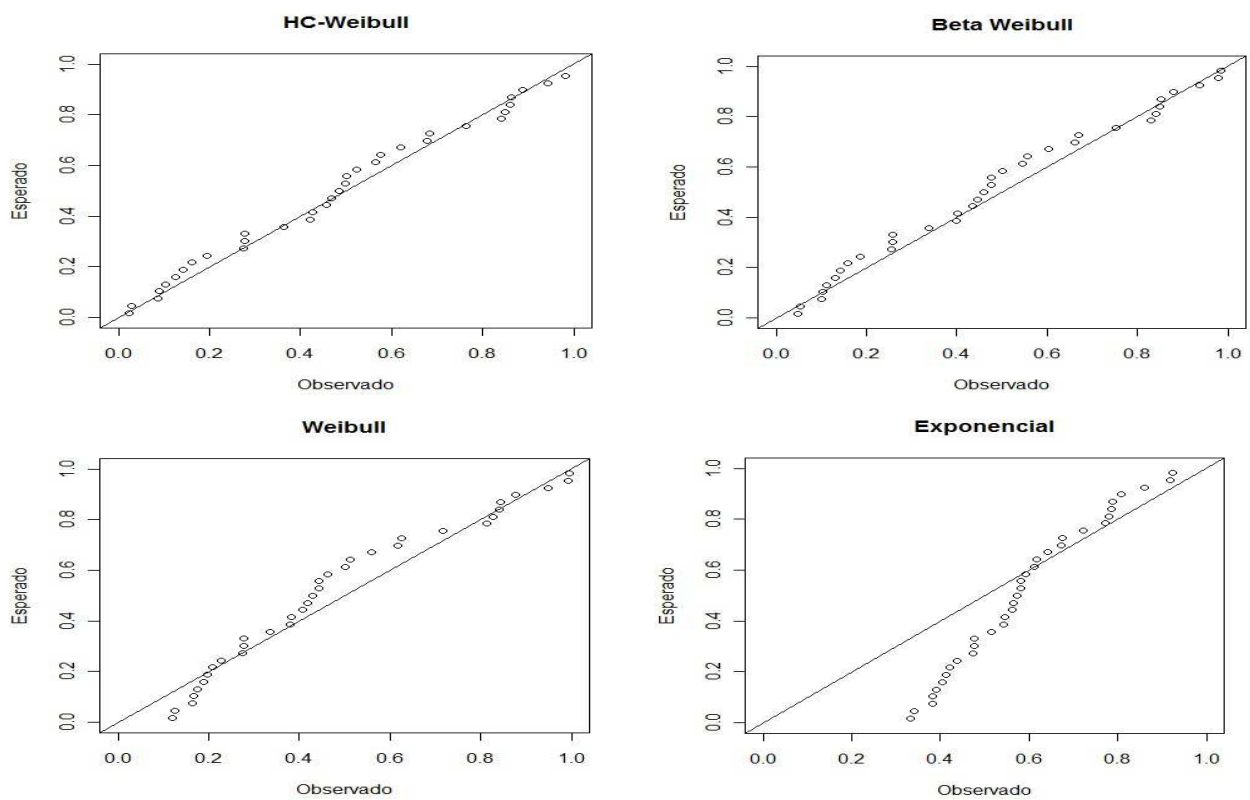

Figura 8: Gráficos de probabilidade para as densidades ajustadas.

\section{Conclusões}

Utilizando os trabalhos de [3], [4] e [17] foi intoduzida uma nova família de distribuições generalizadas. Para cada distribuição G pode-se definir uma nova distribuição,

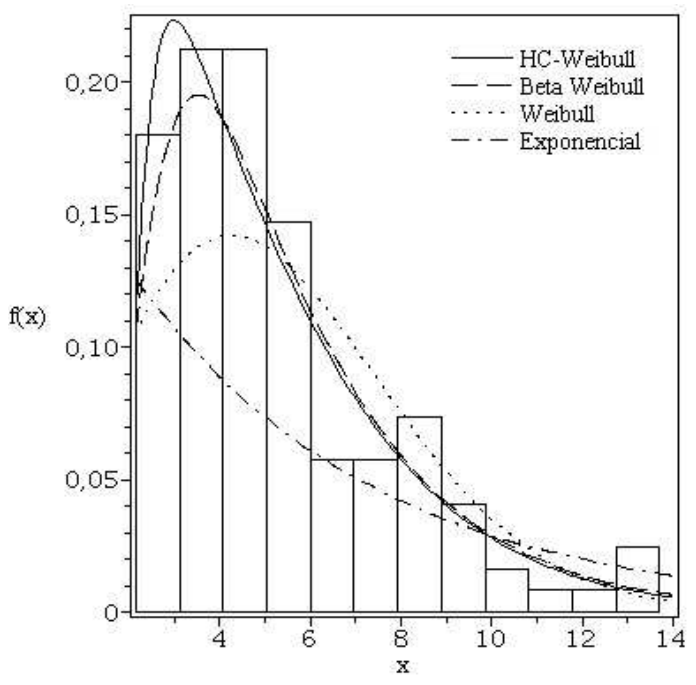

Figura 9: Histograma dos dados observados sobreposto pelas densidades ajustadas. 
hipergeométrica confluente $\mathrm{G}$, que a generaliza utilizando uma fórmula simples.

A estimação dos parâmetros dessa nova família pelo Método da Máxima Verossimilhança pode ser facilmente computada utilizando métodos numéricos. Uma aplicação da distribuição hipergeométrica confluente Weibull a dados reais demonstrou a flexibilidade e o potencial da família hipergeométrica confluente $\mathrm{G}$ na análise de dados de sobrevivência.

Abstract. In this paper we introduced a family of distributions called confluent hypergeometric $\mathrm{G}$ that includes the important models beta normal, beta Weibull, beta Gumbel, beta Pareto among others. New distributions are presented as members of this family, for example, confluent hypergeometric normal distribution and confluent hypergeometric Weibull distribution. The estimation of the parameters of this new class of generalized distributions is studied using the maximum likelihood method and its potential is demonstrated in the modeling of a real data set of thirty-five children with growth hormone deficiency.

Keywords. Generalized beta distribution, confluent hypergeometric distribution, method of maximum likelihood.

\section{Referências}

[1] A. Akinsete, F. Famoye, C. Lee, The beta-Pareto distribution, Statistics, 42, No. 6 (2008), 547-563.

[2] A. Choudhury, A simple derivation of moments of the exponentiated Weibull distribution, Metrika, 62, No. 1 (2005), 17-22.

[3] G.M. Cordeiro, M. Castro, A new family of generalized distributions, J. Stat. Comp. Simulation, 81, No. 7 (2011), 883-898.

[4] N. Eugene, C. Lee, F. Famoye, Beta-normal distribution and its application, Commun. Stat. - Theory and Methods, 31 (2002), 497-512.

[5] M.B. Gordy, Computationally convenient distributional assumptions for common-Value auctions, Comp. Econom., 12, No. 1 (1998), 61-78.

[6] M.S. Khan, The beta inverse Weibull distribution, Int. Trans. Math. Sciences and Comp., 3, No. 1 (2010), 113-119.

[7] C. Lee, F. Famoye, O. Olumolade, Beta-Weibull distribution, J. Mod. Appl. Stat. Meth., 6, No. 1 (2007), 173-186.

[8] A.L. Morais, "A class of generalized beta distributions, Pareto power series and Weibull power series", Dissertação para a obtenção do grau de Mestre em Estatística, Universidade Federal de Pernambuco, Recife, PE, 2009.

[9] G.S. Mudholkar, D.K. Srivastava, M. Friemer, M. Castro, The exponentiated Weibull family: A reanalysis of the bus-motor-failure data, Technometrics, 37 (1995), 436-445. 
[10] S. Nadarajah, A.K. Gupta, On the moments of the exponentiated Weibull distribution., Commun. Stat. - Theory and Methods, 34, No. 2 (2005), 253-256.

[11] S. Nadarajah, S. Kotz, The beta Gumbel distribution, Math. Prob. Eng., 10 (2004), 323-332.

[12] S. Nadarajah, S. Kotz, The beta exponential distribution, Reliab. Eng. and Syst. Safety, 91 (2006), 689-697.

[13] M.M. Nassar, F.H. Eissa, On the exponentiated Weibull distribution, Commun. Stat. - Theory and Methods, 32, No. 7 (2003), 1317-1336.

[14] K. Oldham, J. Myland, J. Spanier, "An atlas of functions: with equator, the atlas function calculator", Springer Vergland, Canadá, 2009.

[15] M. Pal, M.M. Ali, J. Woo, Exponentiated Weibull distribution, Statistica, 66, No. 2 (2006), 139-147.

[16] P.F. Paranaíba, E.M.M. Ortega, G.M. Cordeiro, R.R. Pescim, The beta Burr XII distribution with application to lifetime data, Commun. Stat. - Theory and Methods, 55, No. 2 (2011), 1118-1136.

[17] M.A.R. de Pascoa, E.M.M. Ortega, G.M. Cordeiro, The Kumaraswamy generalized gamma distribution with application in survival analysis, Commun. Stat. - Theory and Methods, 8, No. 5 (2011), 411-433.

[18] K. Persson, J. Ryden, Exponentiated Gumbel distribution for estimation of return levels of significant wave height, J. Envir. Stat., 1, No. 3 (2010), 1-12.

[19] G.O. Silva, E.M.M. Ortega, G.M. Cordeiro, The beta modified Weibull distribution, Math. and Stat., 16, No. 3 (2010), 409-430. 